\title{
Ciclo de Mejora en el Aula (CIMA) en la Parte Práctica de la Asignatura de Proyectos. Anteproyecto de una almazara en Arahal (Sevilla)
}

\section{Classroom Improvement Cycle in the Practical Part of the Project Subject. Preliminary draft of an oil mill in Arahal (Seville)}

MIGUEL ÁNGEL ZARCO GARRIDO

ORCID: https://orcid.org/0000-0002-1455-9468

Universidad de Sevilla

Departamento de Ingeniería de la

Construcción y Proyectos de Ingeniería

mzarco@us.es

Fecha de recepción:

Fecha de aceptación:

DOI: http://dx.doi.org/10.12795/9788447221912.029

Pp.: 665-691 


\section{Resumen}

El Ciclo de Mejora en el Aula (CIMA) ha tenido lugar en la parte Práctica de la asignatura de Proyectos del cuarto curso de las titulaciones del Grado en Ingeniería Química (GIQ) y del Grado en Ingeniería de Tecnologías Industriales (GITI) impartidas en la Escuela Técnica Superior de Ingenieros de la Universidad de Sevilla durante el primer cuatrimestre del curso académico 2019/2020. En la Parte Práctica de esta asignatura se redacta (en grupo) un proyecto de Ingeniería Industrial con un alcance similar a un anteproyecto o proyecto básico, que eligen los alumnos.

Palabras Clave: Ciclo de Mejora en el Aula (CIMA), Asignatura de Proyectos, Grado en Ingeniería Química, Grado en Ingeniería de Tecnologías Industriales, Docencia Universitaria, Experimentación Docente Universitaria.

\section{Abstract}

The Cycle of Improvement in the Classroom has taken place in the Practical Part of the subject of Projects of the fourth course of the degrees of the Degree in Chemical Engineering and the Degree in Engineering of Industrial Technologies taught in the Higher Technical School of Engineers of the University of Seville during the first semester of the 2019/2020 academic year. In the Practical Part of this subject, an Industrial Engineering project is drafted (in groups) with a scope similar to a basic project or draft, which students choose.

Keywords: Classroom Improvement Cycle, Project Subject, Degree in Chemical Engineering, Degree in Industrial Technology Engineering, University Teaching, University Teaching Experimentation.

Jornadas de Formación e Innovación Docente del Profesorado | № 2 (2019) 


\section{Descripción del contexto}

Los alumnos, que son 20, tienen distintas especialidades o intensificaciones: Procesos químicos, Ingeniería Ambiental, Tecnología Industrial, Mecánica-Máquinas, Mecánica-Construcción, Materiales, Energética, Eléctrica, Automática y Organización y Producción. Por ello, el anteproyecto debe tener contenido para todas ellas; ya que el trabajo de la asignatura, que se realiza en grupo, se realiza a su vez en subgrupos, tantos como especialidades hay en clase, y cada uno desarrolla y redacta la parte del anteproyecto propia de su especialidad.

La asignatura la enfoco como si estuviese en la vida laboral real. Como si yo tuviese que redactar un anteproyecto que me han encargado (el que han elegido los alumnos) y que el equipo del que dispongo para realizarlo son los propios alumnos. Intento que ellos se impliquen desde el primer momento ya que son los que van a realizarlo. El trabajo del anteproyecto lo hacen los alumnos fuera del horario de clase, trabajando individualmente y/o en grupos de especialidad, interactuando entre ellos dentro de los grupos de especialidad e interactuando los distintos grupos entre sí. Las clases, que se imparten en una sesión semanal de dos horas, equivaldrían a las reuniones de trabajo de seguimiento (reales) del equipo de redacción del anteproyecto. (Zarco, 2018).

\section{Conexión con el proceso previo}

La experimentación docente universitaria anterior y con la que el CIMA que he realizado tiene conexión y continuidad es el segundo CIMA que realicé durante el desarrollo del Curso General de Docencia Universitaria 5 (CGDU5) en el primer cuatrimestre del curso académico 2018/2019. Tuvo lugar en la misma asignatura y grados del presente CIMA. De dicha experiencia docente envié comunicación a

Jornadas de Formación e Innovación Docente del Profesorado | № 2 (2019)

Esta obra se distribuye con la licencia Creative Commons 
las V Jornadas de Formación e Innovación Docente del Profesorado de la Universidad de Sevilla. (Zarco, 2018).

Dicho CIMA tuvo una duración de 6 horas y trató sobre aquellas partes del anteproyecto ("Otros anejos a la Memoria, Documento Planos y Documento Presupuesto") que se estaban redactando en ese momento. En general el resultado fue satisfactorio ya que se consiguieron los objetivos.

\section{Diseño previo del CIMA}

Partiendo de la experiencia anterior y manteniendo los aspectos de la misma y los principios didácticos que la guiaron, y que considero que deben permanecer en el futuro, he diseñado el CIMA que se caracteriza por:

- He mantenido el mismo Modelo Metodológico ya que funcionó muy bien.

- El Mapa de Contenidos y Problemas/Casos es mayor y más completo que el del CIMA anterior ya que abarca la asignatura completa.

- He diseñado una secuencia de actividades para la asignatura completa muy similares a las del anterior CIMA pero que abarca la asignatura completa.

- Para el seguimiento de la evolución de los modelos mentales de los estudiantes, es decir, su aprendizaje, utilizo los cuestionarios inicial y final y a partir de ellos elaboro las Escaleras de Aprendizaje del Grupo y una Tabla de Avances Individuales.

- Para la evaluación durante el proceso de enseñanza aprendizaje utilizo el portafolio.

- La evaluación del docente y del diseño didáctico la realizo mediante el diario del profesor y las encuestas de opinión de los estudiantes.

Jornadas de Formación e Innovación Docente del Profesorado | № 2 (2019)

Esta obra se distribuye con la licencia Creative Commons 
Para su diseño me he basado en los conceptos: Reconfigurar el aula: quitar a la profesora del centro y Poner los alumnos a trabajar del libro: Dar clase con la boca cerrada (Finkel, D. 2008). Se trata de un modelo metodológico que requiere de un alto grado de compromiso, dedicación y responsabilidad por parte de los alumnos y en el que el alumno es el protagonista. En él se distinguen cinco actividades que se van desarrollando de manera cíclica semanal tal y como se indica en la siguiente figura:

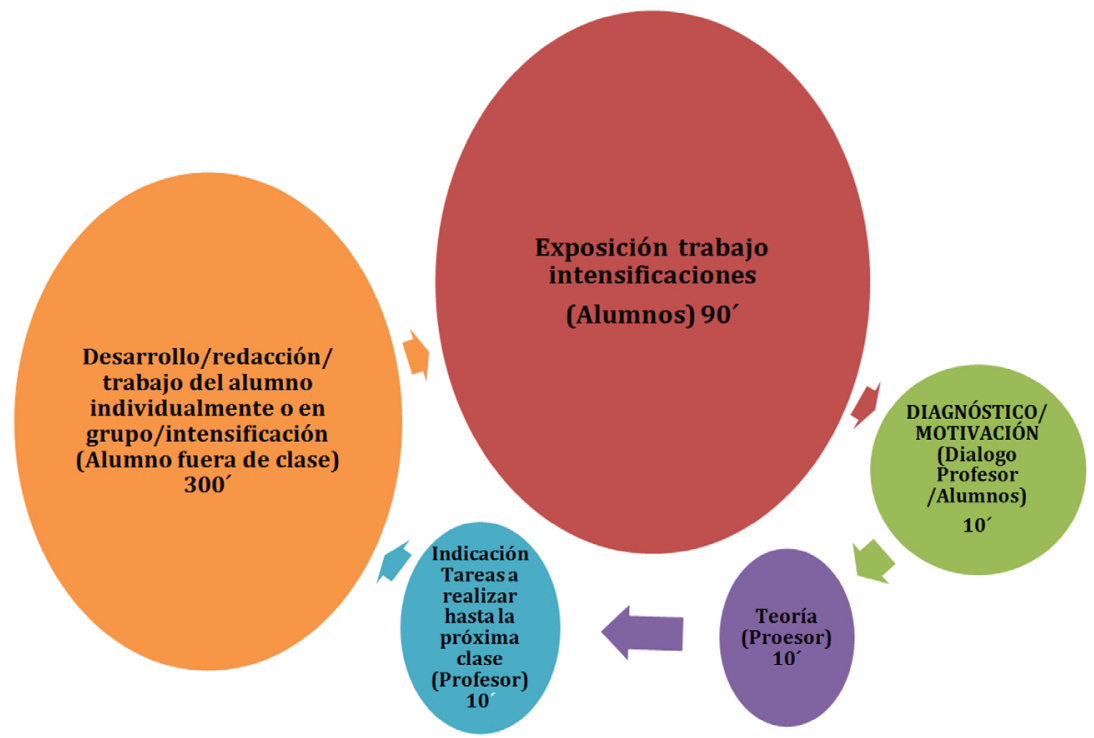

Figura 1. Modelo Metodológico posible

\section{Mapa o red de contenidos o problemas/casos}

Al ser el CIMA de asignatura completa y ser el objeto de la asignatura el desarrollo y la redacción (en grupo) de un proyecto de Ingeniería Industrial con un alcance similar a un anteproyecto o proyecto básico, en el CIMA se trabajan en bloques todas las partes o documentos de las que consta un anteproyecto (Memoria y Anejos a la

Jornadas de Formación e Innovación Docente del Profesorado | № 2 (2019)

Esta obra se distribuye con la licencia Creative Commons Reconocimiento-NoComercial-SinObraDerivada Internacional (CC BY-NC-ND 4.0.) 
Memoria, Documento Planos y Documento Presupuesto): cuáles son, para qué sirven, cómo se redactan y las relaciones entre ellos. Además de lo anterior y previamente a ello se define en qué consiste un anteproyecto y se elige el anteproyecto a redactar teniendo en cuenta que debe tener contenido para todas las especialidades presentes en clase. Teniendo en cuenta todo lo anterior, se ha definido el siguiente Mapa de Contenidos y Problemas en el que se indican los contenidos conceptuales, procedimentales y actitudinales que considero esenciales, sustantivos y organizadores del ciclo y las relaciones más significativas entre ellos, de manera que constituyan un armazón potente de conocimientos básicos.

Se incluye a continuación el Mapa de Contenidos y Problemas diseñado en el que se distinguen 6 bloques de contenidos/problemas, siendo dos semanas la duración de cada uno de ellos:

Jornadas de Formación e Innovación Docente del Profesorado | № 2 (2019)

Esta obra se distribuye con la licencia Creative Commons 


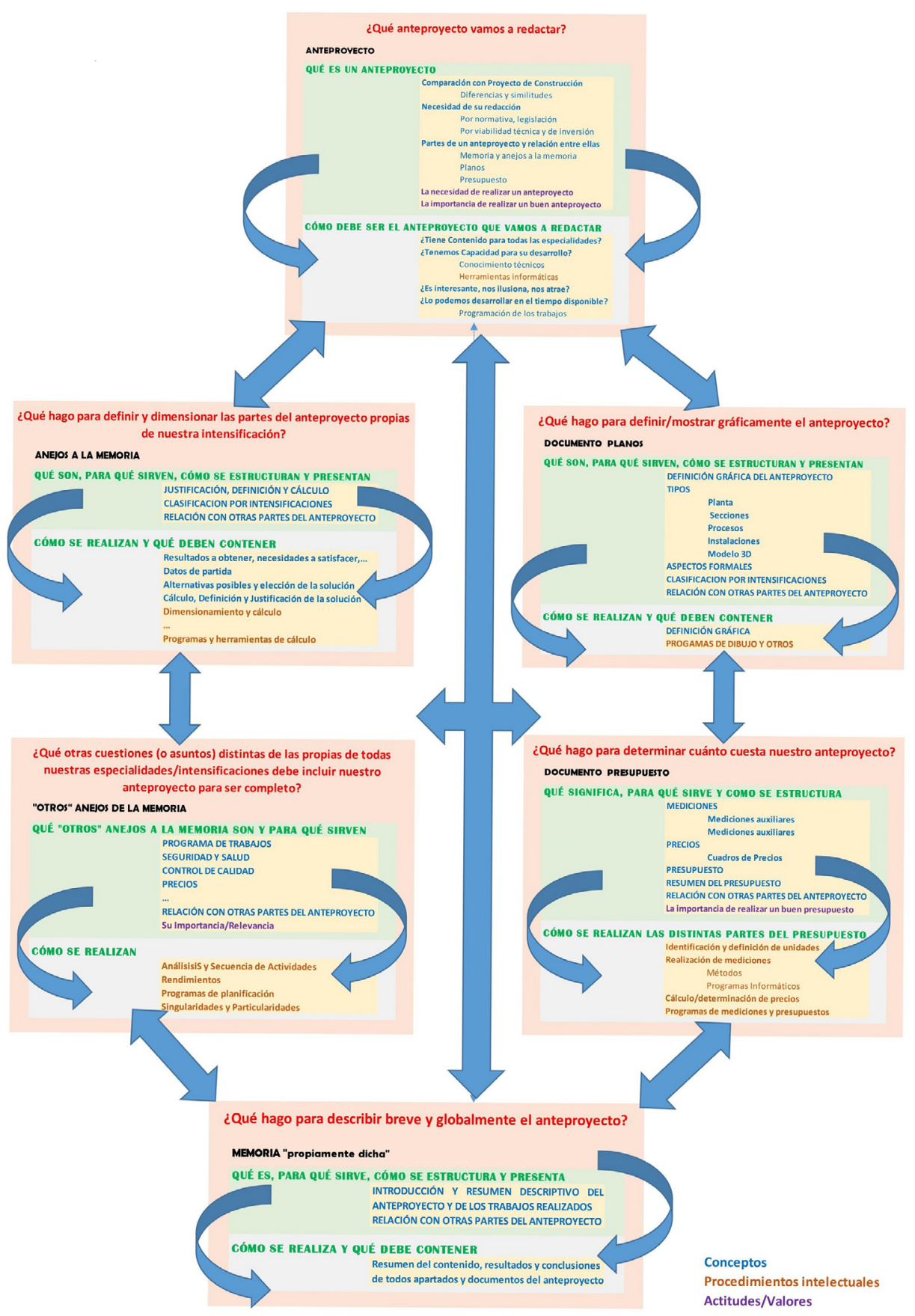

Figura 2. Mapa de Contenidos Problemas

Jornadas de Formación e Innovación Docente del Profesorado | № 2 (2019) 
Se indican a continuación las fichas de actividades programadas para cada uno de los bloques de contenidos del CIMA.

Primer bloque de contenidos: ¿Qué Anteproyecto vamos a redactar? Elección del Anteproyecto.

Tabla 1. Secuencia de actividades del primer bloque de contenidos del CIMA

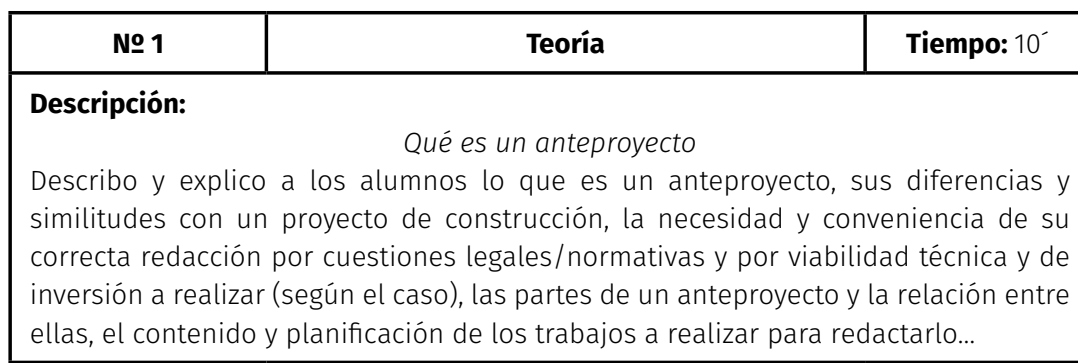

Recursos: Intervención del profesor. Esquemas que se van construyendo/ desarrollando en la pizarra. Ejemplos reales de anteproyectos

\begin{tabular}{|c|c|c|}
\hline № 2 & $\begin{array}{c}\text { Indicación de tareas a realizar durante la } \\
\text { semana hasta la próxima clase }\end{array}$ & Tiempo: $10^{-}$ \\
\hline \multicolumn{3}{|c|}{$\begin{array}{l}\text { Descripción: } \\
\text { Cómo debe ser el anteproyecto que vamos a redactar. Indicaciones para la próxima } \\
\text { exposición } \\
\text { Les trasmito la idea de que el anteproyecto que elijamos redactar tiene que ser tal } \\
\text { que en él haya contenido (partes a desarrollar) para todas las intensificaciones, que } \\
\text { ellos deben tener capacidad (conocimientos técnicos y herramientas informáticas) } \\
\text { para redactarlo y que se pueda desarrollar en el tiempo disponible para ello (primer } \\
\text { cuatrimestre del curso 2019-2020). } \\
\text { Igualmente les indico que sería muy interesante que el anteproyecto a redactar les } \\
\text { resulte interesante, les atraiga y les ilusione. } \\
\text { Mi objetivo es conseguir que sean conscientes de lo que es un anteproyecto, de } \\
\text { lo que se requiere para redactarlo y de su situación (fortalezas y debilidades del } \\
\text { equipo que lo va a redactar) para que puedan proponer y elegir acertadamente el } \\
\text { anteproyecto a redactar. }\end{array}$} \\
\hline
\end{tabular}

Jornadas de Formación e Innovación Docente del Profesorado | № 2 (2019)

Esta obra se distribuye con la licencia Creative Commons Reconocimiento-NoComercial-SinObraDerivada $\quad 4.0$ Internacional (CC BY-NC-ND 4.0.) 
Interviene el profesor y expone concretamente las tareas que deben realizar los alumnos individualmente o en grupos para la siguiente clase. Se trata de que preparen, propuestas de anteproyecto a redactar en las que expongan y justifiquen la viabilidad de su redacción (que tiene contenido para todas las especialidades, que el grupo tiene capacidad para su desarrollo y que se puede desarrollar en el tiempo disponible).

Recursos: Intervención del profesor. Esquemas que se van construyendo/ desarrollando en la pizarra. Ejemplos reales de anteproyectos

\begin{tabular}{|c|c|c|}
\hline № 3 & $\begin{array}{l}\text { Desarrollo/redacción/ trabajo del alumno } \\
\text { individualmente o en grupo } \\
\text { (Alumno fuera de clase) }\end{array}$ & Tiempo: $300^{-}$ \\
\hline \multicolumn{3}{|c|}{$\begin{array}{l}\text { Descripción: } \\
\text { - Preparación de las propuestas de anteproyecto a redactar } \\
\text { - Los alumnos realizan el trabajo fuera de clase de manera individual o en } \\
\quad \text { grupo. }\end{array}$} \\
\hline \multicolumn{3}{|c|}{ Recursos: Manuales, Aplicaciones informáticas } \\
\hline № 4 & Exposición trabajo & Tiempo: $90^{-}$ \\
\hline \multicolumn{3}{|c|}{ 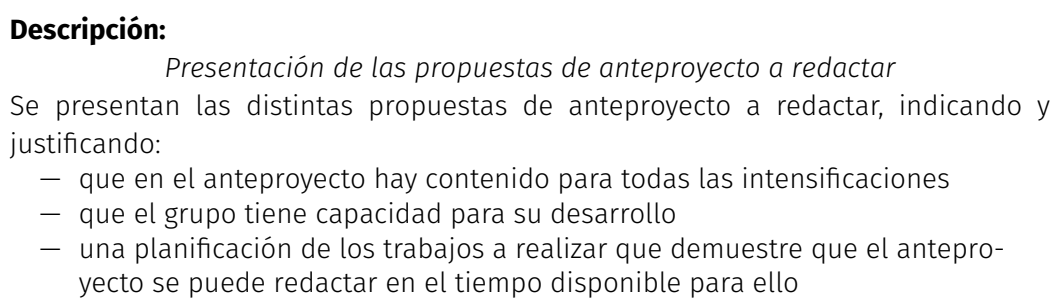 } \\
\hline \multicolumn{3}{|c|}{ Recursos: Presentación de los alumnos con power point } \\
\hline No 5 & Elección del anteproyecto & Tiempo: $10^{-}$ \\
\hline \multicolumn{3}{|c|}{$\begin{array}{l}\text { Descripción: } \\
\qquad \text { Elección del Anteproyecto } \\
\text { A la vista de las presentaciones los alumnos eligen el anteproyecto a redactar. Si es } \\
\text { necesario se procede a una votación entre las opciones consideradas. }\end{array}$} \\
\hline
\end{tabular}

Segundo bloque de contenidos: ¿Qué hago para definir y dimensionar las partes del anteproyecto propias de nuestra intensificación? Anejos a la memoria.

Jornadas de Formación e Innovación Docente del Profesorado | № 2 (2019)

Esta obra se distribuye con la licencia Creative Commons Reconocimiento-NoComercial-SinObraDerivada $\quad 4.0$ Internacional (CC BY-NC-ND 4.0.) 
Tabla 2. Secuencia de actividades del segundo bloque de contenidos del CIMA

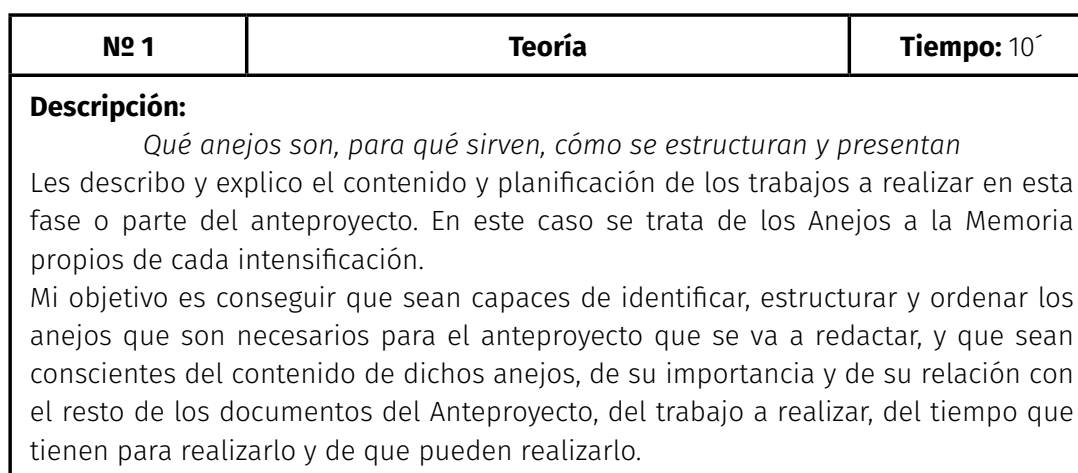

Recursos: Intervención del profesor. Esquemas que se van construyendo/

desarrollando en la pizarra. Ejemplos reales de anteproyectos.

\begin{tabular}{|c|c|c|}
\hline № 2 & & $10^{-}$ \\
\hline \multicolumn{3}{|c|}{$\begin{array}{l}\text { Descripción: } \\
\text { Cómo se realizan y qué deben contener. Indicaciones para la próxima exposición de } \\
\qquad \text { grupos } \\
\text { Interviene el profesor y expone cómo se realizan los Anejos a la Memoria propios de } \\
\text { cada intensificación, qué deben contener y las tareas que deben realizar los alumnos } \\
\text { en grupos para la siguiente clase (resultados a obtener, necesidades a satisfacer, } \\
\text { datos de partida, alternativas posibles y elección de la solución, cálculo, definición y } \\
\text { justificación de la solución, ...). } \\
\text { Cada grupo de especialidad debe presentar al resto la planificación y el avance de } \\
\text { su trabajo. }\end{array}$} \\
\hline
\end{tabular}

Recursos: Intervención del profesor. Esquemas que se van construyendo/ desarrollando en la pizarra. Ejemplos reales de anteproyectos.

\begin{tabular}{|l|c|c|}
\hline \multicolumn{1}{|c|}{ № 3 } & $\begin{array}{c}\text { Desarrollo/redacción/ trabajo del alumno } \\
\text { individualmente o en grupo } \\
\text { (Alumno fuera de clase) }\end{array}$ & Tiempo: $300^{-}$ \\
\hline $\begin{array}{c}\text { Descripción: } \\
\text { Redacción de los anejos a la memoria propios de cada intensificación } \\
\text { Los alumnos realizan el trabajo fuera de clase de manera individual y también en } \\
\text { grupo. }\end{array}$ \\
\hline $\begin{array}{l}\text { Recursos: Publicaciones, Manuales, Catálogos, Normativa y Legislación, Aplicaciones } \\
\text { informáticas }\end{array}$ & \multicolumn{1}{|c|}{ Exposición trabajo de especialidad } & Tiempo: $90^{-}$ \\
\hline \multicolumn{1}{|c|}{ № 4} &
\end{tabular}

Jornadas de Formación e Innovación Docente del Profesorado | № 2 (2019)

Esta obra se distribuye con la licencia Creative Commons Reconocimiento-NoComercial-SinObraDerivada $\quad 4.0$ Internacional (CC BY-NC-ND 4.0.) 
Recursos: Intervención del profesor. Esquemas que se van construyendo/ desarrollando en la pizarra. Ejemplos reales de anteproyectos.

\begin{tabular}{|c|c|c|}
\hline № 2 & $\begin{array}{l}\text { Indicación de tareas a realizar durante } \\
\text { la semana hasta la próxima clase }\end{array}$ & Tiempo: $10^{-}$ \\
\hline \multicolumn{3}{|c|}{$\begin{array}{l}\text { Descripción: } \\
\text { Cómo se realizan. Indicaciones para la próxima exposición de grupos } \\
\text { Interviene el profesor y expone concretamente cómo deben realizar los "Otros" } \\
\text { Anejos a la Memoria y las tareas que deben realizar los alumnos en grupos para la } \\
\text { siguiente clase. }\end{array}$} \\
\hline
\end{tabular}

Recursos: Intervención del profesor. Esquemas que se van construyendo/ desarrollando en la pizarra. Ejemplos reales de anteproyectos.

\begin{tabular}{|c|c|c|}
\hline № 3 & $\begin{array}{l}\text { Desarrollo/redacción/ trabajo del } \\
\text { alumno individualmente o en grupo } \\
\text { (Alumno fuera de clase) }\end{array}$ & Tiempo: $300^{-}$ \\
\hline \multicolumn{3}{|c|}{ Redacción de los "otros" anejos a la memoria } \\
\hline
\end{tabular}

Recursos: Publicaciones, Manuales, Catálogos, Normativa y Legislación, Aplicaciones informáticas

\begin{tabular}{|c|c|c|}
\hline № 4 & Exposición trabajo de especialidad & Tier \\
\hline \multicolumn{3}{|c|}{$\begin{array}{l}\text { Descripción: } \\
\text { Presentación de "otros" anejos a la memoria redactados } \\
\text { Exposición del trabajo realizado y a realizar por los distintos grupos. } \\
\text { Los distintos grupos presentarán lo siguiente: } \\
\text { cómo han desarrollado y cómo van a concluir sus "Otros" Anejos a la Memoria, } \\
\text { mediante un índice o partes a desarrollar que deben explicar brevemente } \\
\text { las dificultades o datos que necesitan y cómo lo han solventado o lo van a solventar } \\
\text { la planificación de trabajos que van a realizar y el compromiso de su cumplimiento. }\end{array}$} \\
\hline \multicolumn{3}{|c|}{ Recursos: Presentación de los alumnos con power point } \\
\hline № 5 & Diagnóstico y Motivación & Tiempo: $10^{-}$ \\
\hline \multicolumn{3}{|c|}{$\begin{array}{l}\text { Descripción: } \\
\text { Diagnóstico/discusión sobre "otros" anejos a la memoria redactados } \\
\text { El profesor a la vista de las exposiciones realizadas diagnostica la situación global } \\
\text { y particular de cada grupo (si está bien enfocado, estructurado y planificado el } \\
\text { trabajo, si el avance es adecuado, ...) e indica las acciones a realizar para corregir las } \\
\text { posibles desviaciones. } \\
\text { El profesor motiva a los alumnos para el alcance de los objetivos. }\end{array}$} \\
\hline & & \\
\hline
\end{tabular}

Jornadas de Formación e Innovación Docente del Profesorado | № 2 (2019)

Esta obra se distribuye con la licencia Creative Commons Reconocimiento-NoComercial-SinObraDerivada $\quad 4.0$ Internacional (CC BY-NC-ND 4.0.) 
Tabla 4. Secuencia de actividades del cuarto bloque de contenidos del CIMA

\begin{tabular}{|c|c|c|}
\hline № 1 & Teoría & Tiempo: $10^{-}$ \\
\hline \multicolumn{3}{|c|}{ Descripción: } \\
\hline \multicolumn{3}{|c|}{$\begin{array}{l}\text { Qué son, para qué sirven los planos (documento planos del anteproyecto), cómo se } \\
\text { estructuran y presentan (aspectos formales) }\end{array}$} \\
\hline \multicolumn{3}{|c|}{$\begin{array}{l}\text { Les describo y explico el contenido y planificación de los trabajos a realizar en la } \\
\text { siguiente fase o parte del anteproyecto. En este caso se trata de los Planos del } \\
\text { Anteproyecto. }\end{array}$} \\
\hline \multicolumn{3}{|c|}{$\begin{array}{l}\text { Mi objetivo es conseguir que sean conscientes del contenido y para qué sirven } \\
\text { los Planos del Anteproyecto, sus tipos, sus aspectos formales, su clasificación por } \\
\text { intensificaciones, su importancia y relación con el resto de los documentos del }\end{array}$} \\
\hline
\end{tabular}

Recursos: Intervención del profesor. Esquemas que se van construyendo/ desarrollando en la pizarra. Ejemplos reales de anteproyectos.

\begin{tabular}{|l|l|l|}
\hline № 2 & $\begin{array}{l}\text { Indicación de tareas a realizar durante la semana } \\
\text { hasta la próxima clase }\end{array}$ & Tiempo: 10` \\
\hline
\end{tabular}

\section{Descripción:}

Cómo se realizan y qué deben contener. Indicaciones para la próxima exposición de grupos

Interviene el profesor y expone concretamente cómo deben realizar los Planos del Anteproyecto y las tareas que deben realizar los alumnos en grupos para la siguiente clase.

Cada grupo de especialidad debe presentar al resto el avance de su trabajo.

Recursos: Intervención del profesor. Esquemas que se van construyendo/ desarrollando en la pizarra. Ejemplos reales de Anteproyectos.

\begin{tabular}{|l|l|l|}
\hline № 3 & $\begin{array}{l}\text { Desarrollo/redacción/ trabajo del alumno } \\
\text { individualmente o en grupo } \\
\text { (Alumno fuera de clase) }\end{array}$ & Tiempo: $300^{\circ}$ \\
\hline $\begin{array}{l}\text { Descripción: } \\
\text { Redacción del documento planos } \\
\text { Los alumnos realizan el trabajo fuera de clase de manera individual y también en } \\
\text { grupo. }\end{array}$ & \multicolumn{2}{|l}{} \\
\hline Recursos: Manuales, Aplicaciones informáticas & Tiempo: $90^{\circ}$ \\
\hline № 4 & Exposición trabajo de especialidad &
\end{tabular}

Jornadas de Formación e Innovación Docente del Profesorado | № 2 (2019)

Esta obra se distribuye con la licencia Creative Commons Reconocimiento-NoComercial-SinObraDerivada $\quad 4.0$ Internacional (CC BY-NC-ND 4.0.) 


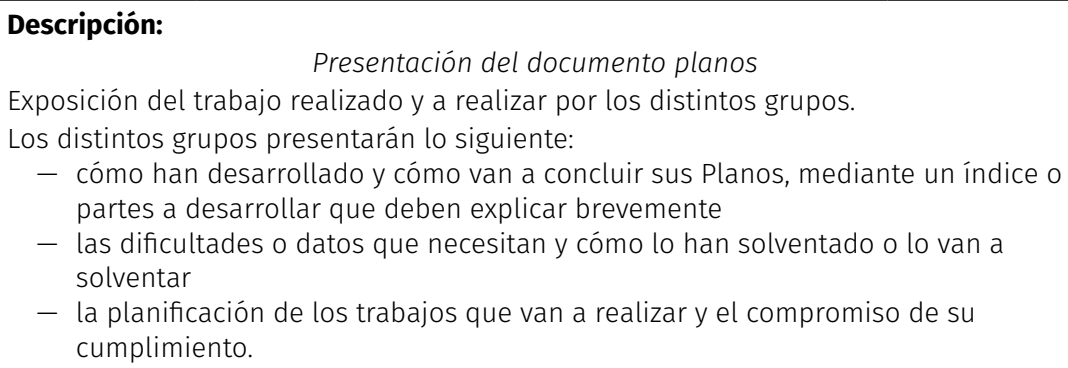

- la planificación de los trabajos que van a realizar y el compromiso de su cumplimiento.

Recursos: Presentación de los alumnos con power point

No 5
$\begin{aligned} & \text { Descripción: } \\ & \text { Diagnóstico/discusión sobre el documento planos presentado }\end{aligned}$
El profesor a la vista de las exposiciones realizadas diagnostica la situación global
y particular de cada grupo (si está bien enfocado, estructurado y planificado el
trabajo, si el avance es adecuado, ...) e indica las acciones a realizar para corregir las
posibles desviaciones.
El profesor motiva a los alumnos para el alcance de los objetivos.

Recursos: Intervención del profesor. Esquemas que se van construyendo/ desarrollando en la pizarra

Quinto bloque de contenidos: ¿Qué hago para determinar cuanto cuesta nuestro anteproyecto? Documento presupuesto.

Tabla 5. Secuencia de actividades del quinto bloque de contenidos del CIMA

\begin{tabular}{|c|c|c|}
\hline № 1 & Teoría & Tiempo: $10^{-}$ \\
\hline \multicolumn{3}{|c|}{$\begin{array}{l}\text { Descripción: } \\
\qquad \text { Qué significa, para qué sirve el presupuesto y cómo se estructura } \\
\text { Les describo y explico el contenido y planificación de los trabajos a realizar en la } \\
\text { siguiente fase o parte del anteproyecto. En este caso se trata del Presupuesto del } \\
\text { Anteproyecto. } \\
\text { Mi objetivo es conseguir que sean conscientes del contenido del Presupuesto del } \\
\text { Anteproyecto, qué significa, para qué sirve, cómo se estructura y su importancia y } \\
\text { relación con el resto de los documentos del Anteproyecto, del trabajo a realizar, del } \\
\text { tiempo que tienen que realizarlo y de que pueden realizarlo. }\end{array}$} \\
\hline \multicolumn{3}{|c|}{$\begin{array}{l}\text { Recursos: Intervención del profesor. Esquemas que se van construyendo/ } \\
\text { desarrollando en la pizarra. Ejemplos reales de anteproyectos. }\end{array}$} \\
\hline № 2 & $\begin{array}{l}\text { Indicación de tareas a realizar durante la semana } \\
\text { hasta la próxima clase }\end{array}$ & Tiempo: $10^{-}$ \\
\hline
\end{tabular}

Jornadas de Formación e Innovación Docente del Profesorado | № 2 (2019)

Esta obra se distribuye con la licencia Creative Commons Reconocimiento-NoComercial-SinObraDerivada $\quad 4.0$ Internacional (CC BY-NC-ND 4.0.) 


\section{Descripción:}

Cómo se realizan las distintas partes del presupuesto. Indicaciones para la próxima exposición de grupos

Interviene el profesor y expone concretamente cómo deben realizar las distintas partes del presupuesto y las tareas que deben realizar los alumnos en grupos para la siguiente clase.

Cada grupo de especialidad debe presentar al resto el avance de su trabajo.

Recursos: Intervención del profesor. Esquemas que se van construyendo/

desarrollando en la pizarra. Ejemplos reales de anteproyectos.

\begin{tabular}{l|l|l|}
\hline № 3 & $\begin{array}{l}\text { Desarrollo/redacción/ trabajo del alumno } \\
\text { individualmente o en grupo/intensificación } \\
\text { (Alumno fuera de clase) }\end{array}$ & Tiempo: 300́ \\
\hline $\begin{array}{l}\text { Descripción: } \\
\text { Redacción de los documentos del presupuesto } \\
\text { Los alumnos realizan el trabajo fuera de clase de manera individual y también en } \\
\text { grupo. }\end{array}$
\end{tabular}

Recursos: Libros, Manuales, Bancos de precios, Aplicaciones informáticas

\begin{tabular}{|c|c|c|}
\hline № 4 & Exposición trabajo de especialidad & Tiempo: $90^{\circ}$ \\
\hline \multicolumn{3}{|c|}{ 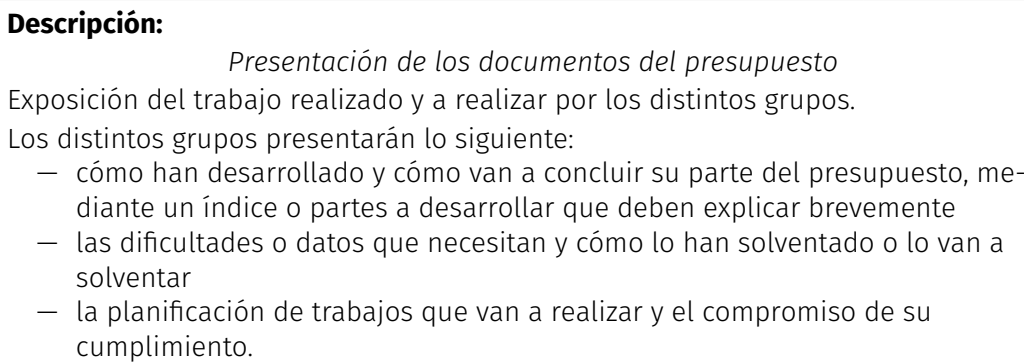 } \\
\hline
\end{tabular}

Recursos: Presentación de los alumnos con power point

\begin{tabular}{|l|l|l|}
\hline № 5 & Diagnóstico y Motivación & Tiempo: 10
\end{tabular}

Descripción:

Diagnóstico/discusión sobre el presupuesto presentado

El profesor a la vista de las exposiciones realizadas diagnostica la situación global y particular de cada grupo (si está bien enfocado, estructurado y planificado el trabajo, si el avance es adecuado, ...) e indica las acciones a realizar para corregir las posibles desviaciones.

El profesor motiva a los alumnos para el alcance de los objetivos.

Recursos: Intervención del profesor. Esquemas que se van construyendo/

desarrollando en la pizarra

Sexto bloque de contenidos: ¿Qué hago para describir breve y globalmente el anteproyecto? Memoria "propiamente dicha".

Jornadas de Formación e Innovación Docente del Profesorado | № 2 (2019)

Esta obra se distribuye con la licencia Creative Commons Reconocimiento-NoComercial-SinObraDerivada $\quad 4.0$ Internacional (CC BY-NC-ND 4.0.) 
Tabla 6. Secuencia de actividades del sexto bloque de contenidos del CIMA

\begin{tabular}{|c|c|c|}
\hline № 1 & Teoría & Tiempo: $10^{-}$ \\
\hline \multicolumn{3}{|c|}{ 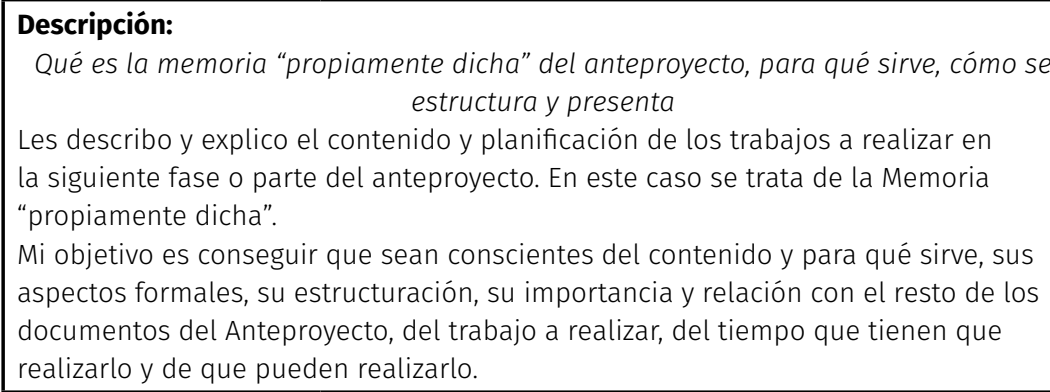 } \\
\hline \multicolumn{3}{|c|}{$\begin{array}{l}\text { Recursos: Intervención del profesor. Esquemas que se van construyendo/ } \\
\text { desarrollando en la pizarra. Ejemplos reales de anteproyectos. }\end{array}$} \\
\hline № 2 & $\begin{array}{l}\text { Indicación de tareas a realizar durante } \\
\text { la semana hasta la próxima clase }\end{array}$ & Tiempo: $10^{-}$ \\
\hline \multicolumn{3}{|c|}{$\begin{array}{l}\text { Descripción: } \\
\text { Cómo se realiza y qué debe contener. Indicaciones para la próxima exposición de } \\
\text { grupos } \\
\text { Interviene el profesor y expone concretamente cómo se debe realizar la memoria } \\
\text { "propiamente dicha” y las tareas que deben realizar los alumnos en grupos para la } \\
\text { siguiente clase. } \\
\text { Cada grupo de especialidad debe presentar al resto el avance de su trabajo. }\end{array}$} \\
\hline \multicolumn{3}{|c|}{$\begin{array}{l}\text { Recursos: Intervención del profesor. Esquemas que se van construyendo/ } \\
\text { desarrollando en la pizarra. Ejemplos reales de anteproyectos. }\end{array}$} \\
\hline № 3 & $\begin{array}{l}\text { Desarrollo/redacción/ trabajo del } \\
\text { alumno individualmente o en grupo } \\
\text { (Alumno fuera de clase) }\end{array}$ & Tiempo: $300^{\prime}$ \\
\hline \multicolumn{3}{|c|}{$\begin{array}{l}\text { Descripción: } \quad \text { Redacción de la memoria "propiamente dicha" } \\
\text { Los alumnos realizan el trabajo fuera de clase de manera individual y también en } \\
\text { grupo. }\end{array}$} \\
\hline \multicolumn{3}{|c|}{ Recursos: Manuales, Aplicaciones informáticas } \\
\hline № 4 & Exposición trabajo de especialidad & Tiempo: $90^{\circ}$ \\
\hline
\end{tabular}

Jornadas de Formación e Innovación Docente del Profesorado | № 2 (2019)

Esta obra se distribuye con la licencia Creative Commons Reconocimiento-NoComercial-SinObraDerivada $\quad 4.0$ Internacional (CC BY-NC-ND 4.0.) 


\section{1}

Jornadas de Formación e Innovación Docente del Profesorado | № 2 (2019)

(c) (i) $(-)$ Esta obra se distribuye con la licencia Creative Commons 


\begin{tabular}{|c|c|c|}
\hline \multicolumn{3}{|c|}{$\begin{array}{l}\text { - cómo hartes a desarrollar que deben explicar brevemente } \\
\text { - las dificultades o datos que necesitan y cómo lo han solventado o lo van a } \\
\text { solventar } \\
\text { - la planificación de los trabajos que van a realizar y el compromiso de su } \\
\text { cumplimiento. }\end{array}$} \\
\hline \multicolumn{3}{|c|}{ Recursos: Presentación de los alumnos con power point } \\
\hline № 5 & Diagnóstico y Motivación & po: $10^{-}$ \\
\hline \multicolumn{3}{|c|}{$\begin{array}{l}\text { Diagnóstico/discusión sobre el documento memoria presentado } \\
\text { El profesor a la vista de las exposiciones realizadas diagnostica la situación global } \\
\text { y particular de cada grupo (si está bien enfocado, estructurado y planificado el } \\
\text { trabajo, si el avance es adecuado, ...) e indica las acciones a realizar para corregir las } \\
\text { posibles desviaciones. } \\
\text { El profesor motiva a los alumnos para el alcance de los objetivos. }\end{array}$} \\
\hline $\begin{array}{l}\text { Recu } \\
\text { desal }\end{array}$ & profesor. Esquemas qu & \\
\hline
\end{tabular}

\section{Cuestionario inicial-final}

Coincidiendo con los problemas-preguntas del mapa de contenidos, el cuestionario inicial-final que se utiliza para hacer el seguimiento de la progresión de los modelos mentales de los estudiantes y de los obstáculos de aprendizaje más comunes que se presentan, es el siguiente:

- Pregunta 1. ¿Qué Anteproyecto vamos a redactar?

- Pregunta 2. ¿Qué hago para definir y dimensionar las partes del anteproyecto propias de nuestra intensificación? Anejos a la memoria.

- Pregunta 3. ¿Qué otras cuestiones (o asuntos) distintas de las propias de todas nuestras especialidades/intensificaciones debe incluir nuestro anteproyecto para ser completo? "Otros" anejos a la memoria.

- Pregunta 4. ¿Qué hago para definir/mostrar gráficamente el anteproyecto? Documento Planos.

Jornadas de Formación e Innovación Docente del Profesorado | № 2 (2019)

Esta obra se distribuye con la licencia Creative Commons 
- Pregunta 5. ¿Qué hago para determinar cuánto cuesta nuestro anteproyecto? Documento Presupuesto.

- Pregunta 6. ¿Qué hago para describir breve y globalmente el anteproyecto? Memoria "propiamente dicha".

El cuestionario inicial se pasa antes de empezar a impartir las clases (el primer día de clase que dedico a presentaciones personales y de la asignatura) para tener algo de tiempo para analizarlo y ajustar el mapa de contenidos y las actividades si fuese necesario. El cuestionario final se pasa al finalizar el CIMA.

\section{Aplicación del CMD}

\section{Relato resumido de sesiones}

Las actividades se desarrollaron con normalidad según lo diseñado y sin variaciones que destacar sobre lo previsto. Los alumnos presentaron tres propuestas de anteproyecto a redactar: una factoría de elementos aeronáuticos, una central de energía renovable productora de hidrógeno y una almazara. Eligieron redactar el anteproyecto de una almazara en Arahal (Sevilla).

Fuera de clase los alumnos trabajaron correctamente preparando las presentaciones y avanzando en la redacción de los distintos documentos del anteproyecto. En clase, los alumnos fueron muy participativos y activos. Ellos mismos, sin necesidad de que el profesor interviniera, tomaban la iniciativa a la hora establecer el orden de intervención de los distintos grupos para que las exposiciones, debate y resolución de cuestiones planteadas en las mismas fuese lo más provechoso y adecuado a sus necesidades. Durante las exposiciones de los alumnos el

Jornadas de Formación e Innovación Docente del Profesorado | № 2 (2019)

Esta obra se distribuye con la licencia Creative Commons 
profesor estaba a un lado de la clase; sólo realizaba intervenciones puntuales cuando veía conveniente y oportuno realizar alguna matización o introducir o recalcar alguna idea. Si el profesor no intervenía, la clase se desarrollaba con normalidad ("iba sola").

Aquellas actividades en las que el peso de las mismas recaía en el profesor (Teoría, Indicación de tareas a realizar durante la semana hasta la próxima clase y Diagnóstico y motivación) se desarrollaron con mucha facilidad ya que los alumnos participaron y preguntaron bastante hasta que las ideas quedaban claras.

Durante el desarrollo de las sesiones me sentí muy bien: por el buen comportamiento y actitud de los alumnos, por su responsabilidad, participación, diligencia, buena relación y comunicación entre ellos, por el gran interés que los alumnos mostraban por el anteproyecto que estaban redactando y por el desarrollo de la asignatura; y sobre todo, porque percibía que el "aprendizaje" se estaba produciendo y porque veía como en clase se materializaban los principios didácticos tenidos en cuenta para el diseño del CIMA: Reconfigurar el aula: quitar al profesor del centro y Poner a los estudiantes a trabajar del libro Dar clase con la boca cerrada (Finkel, D. (2008).

El resultado ha sido muy satisfactorio para mí ya que el CIMA se desarrolló tal y como se diseñó y se consiguieron los objetivos previstos. Pienso que la experiencia también ha sido satisfactoria para los alumnos.

\section{Seguimiento del aprendizaje (de la evolución de los modelos mentales de los estudiantes). Escaleras de Aprendizaje y Tabla de Avances Individuales}

A partir del cuestionario inicial-final he elaborado para cada una de las preguntas del cuestionario inicial-final las Escaleras de Aprendizaje del grupo, que en este caso y por

Jornadas de Formación e Innovación Docente del Profesorado | № 2 (2019)

Esta obra se distribuye con la licencia Creative Commons

Reconocimiento-NoComercial-SinObraDerivada $\quad 4.0$ Internacional (CC BY-NC-ND 4.0.) 
cuestiones de espacio he representado en forma de gráfico, y una Tabla de Avances Individuales con los que he realizado el seguimiento de la evolución de los modelos mentales de los estudiantes, es decir, de su aprendizaje a lo largo del CIMA, para así saber en qué grado mi diseño ha promovido los aprendizajes previstos y si los estudiantes han superado o no los obstáculos detectados.

Siguiendo lo indicado el libro: Enseñanza universitaria. Cómo mejorarla (Porlán, R. 2017), las respuestas, tanto iniciales como finales, las he analizado y las he agrupado según su nivel de complejidad. Estos grupos los he ordenado desde los que representan un menor nivel (respuestas más simples y más alejadas del conocimiento de referencia) hasta los que representan un nivel más alto (respuestas más complejas y más próximas a dicho conocimiento). Los cuatro niveles considerados, de menor a mayor, son:

- A: No responde o responde con ideas muy básicas

- B: Responde con ideas aproximadas

- C: Responde con ideas adecuadas

- D: Respuesta ideal

En las escaleras (grafico) de aprendizaje he indicado, para cada una de las preguntas del cuestionario y tanto al inicio como al final del CIMA, el porcentaje de respuestas de los alumnos que están en cada uno de los cuatro niveles considerados.

En la tabla de avances individuales he indicado para cada uno de los alumnos y para cada una de las preguntas del cuestionario el nivel en el que se encontraba al inicio y al final del CIMA y la progresión experimentada.

Jornadas de Formación e Innovación Docente del Profesorado | № 2 (2019)

Esta obra se distribuye con la licencia Creative Commons 


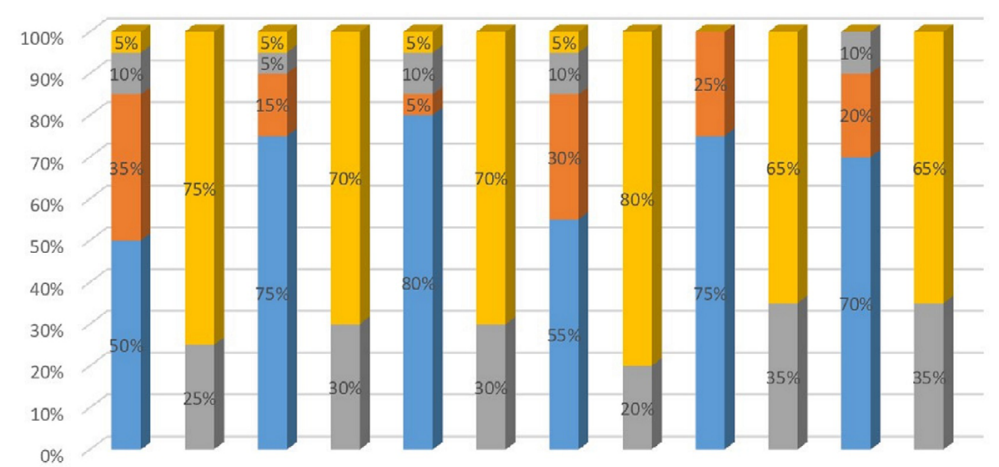

Figura 3. Escaleras (gráfico) de aprendizaje

Analizando la Escalera (gráfico) de aprendizaje se observa que para todas las preguntas se ha pasado de una situación inicial en la que la mayoría los alumnos están en los menores niveles (niveles A y B) a una situación final en la que todos los alumnos están en los mayores niveles (niveles $C$ y $D$ ).

Tabla 7. Tabla de avances individuales

\begin{tabular}{|c|c|c|c|c|c|c|c|c|c|c|c|c|c|c|c|c|c|c|}
\hline \multicolumn{19}{|c|}{ NIVELES DE DESARROLLO DE IDEAS INICIALES Y FINALES } \\
\hline \multirow{3}{*}{ 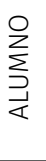 } & \multicolumn{18}{|c|}{ PREGUNTAS } \\
\hline & \multicolumn{3}{|c|}{1} & \multicolumn{3}{|c|}{2} & \multicolumn{3}{|c|}{3} & \multicolumn{3}{|c|}{4} & \multicolumn{3}{|c|}{5} & \multicolumn{3}{|c|}{6} \\
\hline & I & $\mathrm{F}$ & A & I & $\mathrm{F}$ & A & I & $\mathrm{F}$ & A & I & $\mathrm{F}$ & A & I & $\mathrm{F}$ & A & I & $\mathrm{F}$ & A \\
\hline 1 & B & C & +1 & D & D & 0 & D & D & 0 & D & D & 0 & B & D & +2 & C & D & +1 \\
\hline 2 & B & C & +1 & A & D & +3 & A & D & +3 & A & C & +2 & A & D & +3 & C & D & +1 \\
\hline 3 & A & $C$ & +2 & A & $C$ & +2 & A & $C$ & +2 & C & D & +1 & $B$ & D & +2 & A & $D$ & +3 \\
\hline 4 & A & D & +3 & A & C & +2 & A & C & +2 & A & C & +2 & A & D & +3 & A & C & +2 \\
\hline 5 & B & D & +2 & A & $D$ & +3 & A & D & +3 & B & D & +2 & A & D & +3 & B & $D$ & +2 \\
\hline 6 & D & D & 0 & C & D & +1 & $C$ & $D$ & +1 & B & D & +2 & $B$ & D & +2 & B & $D$ & +2 \\
\hline
\end{tabular}

Jornadas de Formación e Innovación Docente del Profesorado | № 2 (2019)

Esta obra se distribuye con la licencia Creative Commons 


\begin{tabular}{|c|c|c|c|c|c|c|c|c|c|c|c|c|c|c|c|c|c|c|}
\hline 7 & $\mathrm{~A}$ & $\mathrm{C}$ & +2 & $\mathrm{~A}$ & $\mathrm{D}$ & +3 & $\mathrm{~A}$ & $\mathrm{D}$ & +3 & $\mathrm{~A}$ & $\mathrm{D}$ & +3 & $\mathrm{~A}$ & $\mathrm{D}$ & +3 & $\mathrm{~A}$ & $\mathrm{D}$ & +3 \\
\hline 8 & $\mathrm{~A}$ & $\mathrm{D}$ & +3 & $\mathrm{~A}$ & $\mathrm{D}$ & +3 & $\mathrm{~A}$ & $\mathrm{D}$ & +3 & $\mathrm{~A}$ & $\mathrm{D}$ & +3 & $\mathrm{~A}$ & $\mathrm{D}$ & +3 & $\mathrm{~A}$ & $\mathrm{D}$ & +3 \\
\hline 9 & $\mathrm{C}$ & $\mathrm{D}$ & +1 & $\mathrm{~B}$ & $\mathrm{D}$ & +2 & $\mathrm{~A}$ & $\mathrm{C}$ & +2 & $\mathrm{C}$ & $\mathrm{D}$ & +1 & $\mathrm{~A}$ & $\mathrm{D}$ & +3 & $\mathrm{~A}$ & $\mathrm{D}$ & +3 \\
\hline 10 & $\mathrm{~A}$ & $\mathrm{D}$ & +3 & $\mathrm{~A}$ & $\mathrm{D}$ & +3 & $\mathrm{~A}$ & $\mathrm{C}$ & +2 & $\mathrm{~A}$ & $\mathrm{C}$ & +2 & $\mathrm{~A}$ & $\mathrm{C}$ & +2 & $\mathrm{~A}$ & $\mathrm{C}$ & +2 \\
\hline 11 & $\mathrm{~A}$ & $\mathrm{C}$ & +2 & $\mathrm{~B}$ & $\mathrm{C}$ & +1 & $\mathrm{~A}$ & $\mathrm{D}$ & +3 & $\mathrm{~A}$ & $\mathrm{C}$ & +2 & $\mathrm{~A}$ & $\mathrm{D}$ & +3 & $\mathrm{~A}$ & $\mathrm{C}$ & +2 \\
\hline 12 & $\mathrm{~B}$ & $\mathrm{D}$ & +2 & $\mathrm{~A}$ & $\mathrm{D}$ & +3 & $\mathrm{~A}$ & $\mathrm{C}$ & +2 & $\mathrm{~A}$ & $\mathrm{D}$ & +3 & $\mathrm{~A}$ & $\mathrm{C}$ & +2 & $\mathrm{~A}$ & $\mathrm{D}$ & +3 \\
\hline 13 & $\mathrm{~B}$ & $\mathrm{D}$ & +2 & $\mathrm{~A}$ & $\mathrm{C}$ & +2 & $\mathrm{~A}$ & $\mathrm{D}$ & +3 & $\mathrm{~B}$ & $\mathrm{D}$ & +2 & $\mathrm{~A}$ & $\mathrm{D}$ & +3 & $\mathrm{~B}$ & $\mathrm{C}$ & +1 \\
\hline 14 & $\mathrm{~A}$ & $\mathrm{D}$ & +3 & $\mathrm{~A}$ & $\mathrm{C}$ & +2 & $\mathrm{~A}$ & $\mathrm{D}$ & +3 & $\mathrm{~B}$ & $\mathrm{D}$ & +2 & $\mathrm{~A}$ & $\mathrm{C}$ & +2 & $\mathrm{~A}$ & $\mathrm{C}$ & +2 \\
\hline 15 & $\mathrm{~B}$ & $\mathrm{D}$ & +2 & $\mathrm{~A}$ & $\mathrm{C}$ & +2 & $\mathrm{~A}$ & $\mathrm{D}$ & +3 & $\mathrm{~A}$ & $\mathrm{D}$ & +3 & $\mathrm{~B}$ & $\mathrm{C}$ & +1 & $\mathrm{~A}$ & $\mathrm{C}$ & +2 \\
\hline 16 & $\mathrm{~A}$ & $\mathrm{D}$ & +3 & $\mathrm{~A}$ & $\mathrm{D}$ & +3 & $\mathrm{~A}$ & $\mathrm{D}$ & +3 & $\mathrm{~A}$ & $\mathrm{D}$ & +3 & $\mathrm{~A}$ & $\mathrm{D}$ & +3 & $\mathrm{~A}$ & $\mathrm{D}$ & +3 \\
\hline 17 & $\mathrm{~A}$ & $\mathrm{D}$ & +3 & $\mathrm{~A}$ & $\mathrm{D}$ & +3 & $\mathrm{~A}$ & $\mathrm{D}$ & +3 & $\mathrm{~B}$ & $\mathrm{D}$ & +2 & $\mathrm{~B}$ & $\mathrm{D}$ & +2 & $\mathrm{~B}$ & $\mathrm{D}$ & +2 \\
\hline 18 & $\mathrm{C}$ & $\mathrm{D}$ & +1 & $\mathrm{~A}$ & $\mathrm{D}$ & +3 & $\mathrm{~A}$ & $\mathrm{D}$ & +3 & $\mathrm{~A}$ & $\mathrm{D}$ & +3 & $\mathrm{~A}$ & $\mathrm{C}$ & +2 & $\mathrm{~A}$ & $\mathrm{D}$ & +3 \\
\hline 19 & $\mathrm{~A}$ & $\mathrm{D}$ & +3 & $\mathrm{~A}$ & $\mathrm{D}$ & +3 & $\mathrm{~B}$ & $\mathrm{C}$ & +1 & $\mathrm{~B}$ & $\mathrm{D}$ & +2 & $\mathrm{~A}$ & $\mathrm{C}$ & +2 & $\mathrm{~A}$ & $\mathrm{C}$ & +2 \\
\hline 20 & $\mathrm{~B}$ & $\mathrm{D}$ & +2 & $\mathrm{~B}$ & $\mathrm{D}$ & +2 & $\mathrm{C}$ & $\mathrm{D}$ & +1 & $\mathrm{~A}$ & $\mathrm{D}$ & +3 & $\mathrm{~A}$ & $\mathrm{C}$ & +2 & $\mathrm{~A}$ & $\mathrm{D}$ & +3 \\
\hline & \multicolumn{10}{|c|}{} & I: INICIAL, F: FINAL, A: AVANCES & & & & & \\
\hline
\end{tabular}

Analizando la Tabla de avances individuales se observa que en todas las preguntas todos los alumnos han subido de nivel (1, 2 ó 3 niveles según el caso), salvo un alumno en la pregunta 1 y otro alumno en las preguntas 2, 3 y 4 que ya estaban en el mayor nivel y se han mantenido.

Visto todo lo anterior, Escaleras (gráfico) de Aprendizaje y Tabla de Avances Individuales, se puede concluir con que el "aprendizaje se ha producido".

Aunque con la Tabla he obtenido la evolución individual de cada estudiante y su nivel al finalizar el CIMA, no trasladaré los resultados en ningún porcentaje a la calificación de la asignatura ya que la calificación de ésta está basada en la calificación del anteproyecto redactado en grupo y en un examen al final de la asignatura.

\section{Evaluación del Estudiante. Portafolio}

Cada alumno ha creado una carpeta (portafolio) en la que ha ido colocando durante el CIMA las presentaciones

Jornadas de Formación e Innovación Docente del Profesorado | № 2 (2019)

Esta obra se distribuye con la licencia Creative Commons 
que ha preparado y ha utilizado en las exposiciones de trabajo de especialidad realizadas en las clases. El análisis de dichos portafolios confirma y está en consonancia con los resultados y evolución del aprendizaje de los estudiantes obtenidos en las Escaleras (gráfico) y Tabla. No usaré el portafolio para la calificación de la asignatura por las mismas razones expuestas.

\section{Evaluación del propio diseño y de mi intervención}

Al final de cada clase he recogido por escrito en el diario del profesor una descripción, un análisis y una valoración de la práctica docente. En general la valoración es positiva y tendré en cuenta lo recogido para futuros diseños y aplicaciones de CIMÁs.

Al finalizar el CIMA he realizado una encuesta de opinión a los estudiantes con las siguientes preguntas: ¿Qué has aprendido? ¿Por qué crees que ha ocurrido? ¿Cómo lo valoras? ¿Qué mantendrías? ¿Qué eliminarías? ¿Qué propuestas de mejoras haces? Del análisis de las respuestas de los estudiantes sólo destaco (aunque tiene una relación indirecta con el CIMA) que los alumnos proponen como mejora que esta parte práctica de la asignatura de proyectos en la que ha tenido lugar el CIMA tenga lugar después de que se imparta la parte teórica de la asignatura. Ello redundaría en un avance más rápido y más profundo en la redacción del anteproyecto, objeto de esta parte práctica de la asignatura de proyectos.

Jornadas de Formación e Innovación Docente del Profesorado | № 2 (2019)

Esta obra se distribuye con la licencia Creative Commons 


\section{Evaluación del CIMA}

\section{Principios Didácticos argumentados que han guiado la experiencia y que considero que deben permanecer en el futuro:}

- Reconfigurar el aula: quitar al profesor del centro y Poner a los estudiantes a trabajar del libro Dar clase con la boca cerrada (Finkel, D. (2008)

- Aportar las ayudas necesarias en el proceso de aprendizaje del alumno (mediante actividades y recursos especificos) para orientar la transición desde los modelos propios del alumno a los propios de la disciplina, Conocer y analizar los modelos mentales del alumno mediante los cuestionarios y escaleras de aprendizaje, La evaluación del estudiante durante el proceso de enseñanza-aprendizaje mediante el portafolio o carpetas de aprendizaje y La evaluación del docente y del diseño didáctico durante el proceso mediante el diario del profesor y las encuestas de opinión del libro Enseñanza Universitaria. Cómo mejorarla (Porlán, R. 2017)

\section{Cuestiones a mantener y cambios a introducir para un futuro CIMA}

Teniendo en cuenta los Principios Didácticos anteriores, considero que el CIMA se ha desarrollado de manera satisfactoria tanto para los alumnos como para el docente, que el "aprendizaje se ha producido". Por otro lado tendré en cuenta las ideas obtenidas del Curso Específico que he realizado en este primer cuatrimestre "Los mapas de contenidos y problemas: otra manera de diseñar la enseñanza" del programa FIDOP de la Universidad de Sevilla. Por tanto para un futuro CIMA:

Jornadas de Formación e Innovación Docente del Profesorado | № 2 (2019) 
- Mantendré el modelo metodológico ya que ha funcionado muy bien.

- Intentaré mejorar y evolucionar el Mapa de Contenidos y Problemas con los conocimientos adquiridos en el Curso Específico indicado anteriormente.

- Diseñaré una secuencia de actividades similar a la de este CIMA, pero adaptada a los bloques de contenidos del nuevo mapa.

- Diseñaré los cuestionarios inicial y final teniendo en cuenta los contenidos del nuevo mapa de contenidos y elaboraré a partir de ellos las Escaleras de Aprendizaje del grupo y la Tabla de Avances Individuales con los que realizaré el seguimiento de los modelos mentales de los estudiantes (su aprendizaje).

- Utilizaré el portafolio para la evaluación del estudiante durante el proceso de enseñanza aprendizaje que se produzca durante el CIMA.

- Usaré el diario del profesor y realizaré encuestas de opinión para la evaluación del docente y del diseño didáctico del CIMA.

Jornadas de Formación e Innovación Docente del Profesorado | № 2 (2019)

Esta obra se distribuye con la licencia Creative Commons 


\section{Referencias Bibliográficas}

Finkel, D. (2008). Dar clase con la boca cerrada. Valencia: Publicaciones Universidad de Valencia.

Porlán, R. (2017). Enseñanza universitaria. Cómo mejorarla. Madrid: Ediciones Morata, S.L.

Zarco, M.A. (2018). Ciclo de Mejora Docente en la Parte Práctica de la Asignatura de Proyectos. Anteproyecto de una Fábrica de Cerveza. Monográfico Jornadas de Formación e Innovación Docente del Profesorado 1, pp. 1035-1052.

Jornadas de Formación e Innovación Docente del Profesorado | № 2 (2019) Esta obra se distribuye con la licencia Creative Commons Reconocimiento-NoComercial-SinObraDerivada 